\title{
SIX LEVIERS POUR REVALORISER L'IMAGE DE LA LOGISTIQUE
}

\author{
GILLES PACHÉ \& RUDOLF O. LARGE
}

Publié dans Management \& Datascience, le 10 mars 2021

Catégorie : Avis d'expert

DOI : https://doi.org/10.36863/mds.a.16219

RÉSUMÉ

Essentielle au fonctionnement des échanges, et largement mise en lumière pendant la crise sanitaire de la Covid-19, la logistique est loin d'être reconnue à sa réelle valeur. Son image est certainement à améliorer, notamment aux yeux des étudiantes et étudiantes en sciences de gestion et du management, en jouant sur plusieurs leviers d'action. L'article suggère la mise en œuvre, a priori aisée, de six leviers pour revaloriser l'image d'une fonction support de première importance, et $y$ attirer les meilleurs talents dans le futur.

\begin{tabular}{l|l|l|l|l} 
COVID-19 & ETUDIANTS & IMAGE & LOGISTIQUE & MARKETING
\end{tabular}

Citation : Paché, G., \& Large, R. (Mar 2021). Six leviers pour revaloriser l'image de la logistique. Management et Datascience, Article 0016219. https://doi.org/10.36863/mds.a.16219.

Les auteurs:

- Gilles Paché

(gilles.pache@univ-amu.fr) - (Pas d'affiliation)

- Rudolf O. Large

(Pas d'affiliation)

Copyright : @ 2021 les auteurs. Publication sous licence Creative Commons CC BY-ND.

Liens d'intérêts : Le ou les auteurs déclarent ne pas avoir connaissance de conflit d'intérêts impliqués par l'écriture de cet article.

Financement : Le ou les auteurs déclarent ne pas avoir bénéficié de financement pour le travail mis en jeu par cet article.

\section{TEXTE COMPLET}

Il est toujours surprenant - et décevant - de constater que la logistique, une fonction jugée pourtant essentielle dans le fonctionnement des sociétés modernes, soit négligée, et parfois même méprisée par certains (Paché, 2021). En effet, sans elle, il serait impossible de consommer sans entraves, notamment des produits de première nécessité. La crise sanitaire de la Covid-19 a ainsi permis de constater l'existence d'une « urgence logistique » au niveau de l'organisation au jour le jour des systèmes de distribution et d'approvisionnement, à la fois pour les magasins physiques, pour les magasins virtuels et pour les plateformes de livraison à domicile. Grâce à une logistique performante, portée par des femmes et des hommes emplis d'abnégation pendant les différentes périodes de confinement, les consommateurs ont pu continuer à acheter en ligne des produits alimentaires et culturels et se faire livrer des repas à domicile.

II faut le reconnaître : la logistique d'entreprise est peut-être à un moment clé de sa récente histoire. Une crise sanitaire et sociale d'une intensité inconnue depuis la Seconde Guerre mondiale a permis de souligner combien elle est capitale pour permettre aux économies de marché de répondre aux besoins des citoyens. Ce n'est pas une véritable découverte pour les spécialistes du sujet, mais une sorte de révélation pour beaucoup d'individus. Il ne faut donc pas perdre de temps et afin d'attirer les meilleurs talents dans les formations en logistique, un travail d'amélioration de l'image de la logistique est indispensable, alors qu'elle a longtemps été sous-estimée par rapport au marketing et à la finance de marché. II s'agit d'une question ancienne abordée il y a près de vingt ans de cela par Knemeyer \& Murphy (2004), mais qui reste d'actualité. Six leviers peuvent être ici suggérés, qui seront notamment utiles pour les entreprises spécialisées dans la prestation de services logistiques et dans la distribution des produits de grande consommation. 


\section{Bien identifier les bénéfices recherchés par les clients}

Il est purement et simplement suicidaire de proposer à des clients un faible niveau de service logistique alors qu'ils attendent impatiemment de recevoir un produit ou, à l'inverse, de dépenser des sommes exorbitantes pour un niveau de service logistique élevé alors que les clients ne sont pas vraiment pressés. Une logistique aura d'autant plus une excellente image auprès des clients qu'elle fournit exactement le service adapté aux besoins : ni plus, ni moins. Avec un paradoxe très connu : quand un client programme l'arrivée d'un produit commandé en ligne à son domicile le mercredi matin entre $10 \mathrm{~h}$ et $12 \mathrm{~h}$, le livrer le mardi matin pourrait générer une paradoxale non-satisfaction... malgré un délai de livraison raccourci de $24 \mathrm{~h}$, tout simplement parce qu'il est absent de chez lui le mardi ! Pour parvenir à la juste prestation, il est indispensable pour les entreprises d'étudier précisément quelles sont les attentes des clients, par exemple en fonction de situations singulières d'achat (Halley, 1968).

En d'autres termes, une identification des avantages (bénéfices) recherchés par les clients s'avère ici essentielle, ce qui renvoie à une réflexion préalable de nature marketing, que Vernette (1989) a été l'un des premiers chercheurs à populariser en France. Ainsi, un délai de livraison de quelques heures peut être un avantage recherché majeur exprimé par des parents pour répondre à des besoins urgents, par exemple quand la veille de Noël, leur petit garçon attend impatiemment le jouet tant espéré (et promis par le gentil bonhomme habillé en rouge). Ce même délai ne sera pas nécessairement un avantage recherché quand un papy veut spontanément offrir à sa petite-fille Leia une peluche géante, sans raison précise, sinon lui faire un petit plaisir qui pourra attendre quelques jours sans provoquer d'effet déceptif majeur. La juste prestation en point de mire, semaine après semaine, voilà sans doute le plus grand défi à relever, qui n'est pas sans rappeler en clin d'œil le « juste écart » postconfinement de Rouquet (2020).

\section{Offrir une parfaite traçabilité des livraisons}

Il est fort probable que des clients soient moins gênés par le retard de livraison proprement dit que par l'incertitude relative pesant sur le moment de la livraison luimême (Rao et al., 2011). L'image parfois catastrophique que peut avoir la logistique est souvent liée aux délais annoncés aux clients, mais non respectés, surtout en situation d'urgence de consommation, sans qu'il soit possible de disposer d'une information fiable sur l'avancée (plus lente que prévu) de la livraison. De nombreux distributeurs en ligne ont ainsi compris que la récupération ultérieure de clients mécontents, connue sous le nom de « service recovery strategies " (DeWitt \& Brady, 2003), passe par un suivi en temps réel du statut de leur commande, avec un éventuel retard annoncé le plus rapidement possible. Dans ce cas, bénéficiant d'un système d'information ad hoc, les clients auront tendance à moins tenir le vendeur pour responsable de la défaillance logistique.

D'une certaine façon, on peut ici parler d'un empowerment offert au client, qui aura l'impression agréable de contrôler la situation (logistique) plutôt que de la subir passivement. Par exemple, dans le cas d'une commande en ligne, il pourra prendre une décision d'ajustement de son comportement si une défaillance logistique lui est annoncée très en amont de la livraison. Au-delà du simple suivi de la commande en cours, les entreprises ont intérêt à mettre à la disposition des clients une plateforme ergonomique de consultation, de suivi et d'annulation de la commande en fonction des conditions réelles, et non prévisionnelles, de son acheminement. En d'autres termes, les clients doivent être capables d'accéder au système de traçabilité de manière très simple et peu coûteuse, obligeant au demeurant les entreprises à maîtriser parfaitement les données massives pour mener à bien leur politique marketing, ce qui a été maintes fois souligné par ailleurs (Zaïdi-Chtourou, 2018).

\section{Simplifier la politique de retour}

Un client pourra se sentir plus ou moins « piégé " par un site Internet si les conditions logistiques de retour des produits, en cas d'insatisfaction ressentie, sont jugées trop complexes à gérer (Nasir, 2004), et l'obligent à un effort d'organisation personnelle, par exemple se déplacer jusqu'à un bureau de poste ou un point-relais. Sera-t-il prêt alors à prendre le risque d'acheter ? De ce point de vue, il est absolument essentiel pour une entreprise d'agir dans deux directions complémentaires pour éviter des pertes potentielles de vente. D'une part, avant la commande, il s'agira de clarifier les processus et les conditions de retour des produits, afin d'éviter les mauvaises surprises. D'autre part, après la commande, il s'agira de mettre en œuvre les procédures les plus simples possibles - en matière d'efforts consentis par le client - pour faciliter la logistique du retour des produits.

L'idée clé est de transformer un échec commercial relatif, par exemple avec un produit réel dont le client constate qu'il est très décevant par rapport à son exposition virtuelle sur le site Internet (qui n'a jamais réceptionné des chaussures ou une robe dont les atours sont finalement bien différents de ceux espérés ?), en une éclatante réussite logistique, avec un « périple de retour " extrêmement simple à gérer (Rajendran et al., 2018). Il reste évidemment à se poser la question de la prise en charge des coûts de retour. Les clients accepteront très difficilement de payer pour renvoyer un produit qui les a déçus ou qui ne correspond pas tout à fait à leurs attentes. Sans aucune hésitation, la gratuité du service logistique de retour apparaît essentielle pour améliorer significativement l'image de sérieux et de rigueur de l'entreprise auprès de ses clients.

\section{Gérer durablement le flux de produits}

Si la qualité de service offerte à la clientèle, tant pour les livraisons et leur suivi que pour les éventuels retours de produits, permet de valoriser l'image de la logistique, cette dernière pourra aussi mettre en avant le fait qu'elle œuvre désormais activement pour le respect de contraintes environnementales. Aux yeux d'une nouvelle génération de plus en plus sensible à la durabilité, dans un monde soumis à des menaces systémiques majeures (pollution massive, changement climatique, etc.), il est fort probable que des démarches de type sustainable supply chains seront perçues de manière positive. Il est incontestable que de telles démarches se multiplient depuis une quinzaine d'années, et donnent lieu à une abondante littérature académique (Koberg \& Longoni, 2019), mais faute d'une communication adaptée, le risque est grand qu'elles soient vues comme une nouvelle déclinaison des pratiques de greenwashing dont les écologistes les plus radicaux sont de féroces contempteurs. 
Parmi les avancées les plus significatives en matière de gestion durable des flux de produits, les réalisations en termes de logistique urbaine durable (Taniguchi, 2014), en connexion directe avec le développement des ventes en ligne et le retour en force du commerce de proximité, sont à souligner. Sur le plan technique, l'utilisation de véhicules de livraison propres et silencieux tend à se multiplier, tandis que sur le plan organisationnel, des entreprises, même en concurrence frontale, apprennent à mutualiser leurs ressources logistiques pour éviter des redondances de moyens de transport et d'entreposage aux impacts environnementaux négatifs. II n'est plus exceptionnel désormais que des tournées soient collectivement planifiées dans l'espace urbain pour réduire l'emprise spatiale et temporelle des systèmes d'acheminement des marchandises dans la ville. Une révolution silencieuse est en cours, et nul étonnement à ce que de nombreuses universités et business schools européennes réputées comme Audencia, Cardiff, Heriot-Watt ou Strathclyde aient saisi l'occasion pour créer des programmes spécialisés de MSc en sustainable supply chain management.

\section{« Dégenrer » des métiers historiquement masculins}

Force est d'admettre que les femmes sont encore peu présentes dans les postes de top management logistique. Selon les pays, il n'est pas rare que les hommes y occupent les trois quarts des emplois, voire plus, ce qui interpelle sur les raisons d'une telle masculinisation : les entreprises ne recrutent-elles pas assez de talents féminins volontairement, ou les talents féminins disponibles ne sont-ils pas suffisamment disponibles, notamment par la faute de programmes universitaires peu attrayants pour les étudiantes, éduquées par ailleurs en référence à des stéréotypes négatifs sur la logistique ? Or, les évolutions récentes relatives au management des chaînes logistiques soulignent l'importance croissante de soft skills fondés sur la communication interpersonnelle et le sens du contact, autant de compétences que l'on retrouve chez les femmes managers. Plus largement, des recherches sur le genre en contexte logistique soulignent combien les personnalités féminines maîtrisent leurs affects lors d'interactions sociales (Ruel et al., 2020), par exemple pendant les processus de négociation de contrats d'externalisation.

L'intégration des femmes dans le top management logistique pourrait ainsi favoriser la rupture avec une ancienne culture machiste qui se manifeste souvent par un style agressif, et que l'on retrouve également dans les achats, une fonction d'ailleurs très proche de la logistique, comme le souligne la recherche de Lawrence et al. (2018). La mise en œuvre de relations plus collaboratives entre les partenaires d'une chaîne logistique est une condition essentielle à l'amélioration du service offert à la clientèle, entre autres pour mieux piloter les interfaces et les interactions entre acteurs, et le développement d'un « dialogue constructif » y joue un rôle clé. En « dégenrant » les métiers de la logistique, les entreprises feraient ainsi la preuve qu'elles impulsent des stratégies sociétales plus inclusives et qu'elles ont pris conscience de dimensions psychologiques importantes dans la gestion de leurs ressources humaines. L'enjeu est de taille car le plafond de verre reste une réalité très présente en logistique (Hoareau et al., 2020).

\section{Théâtraliser la logistique}

La dernière option suggérée, sans doute la plus surprenante et inattendue, est de « mettre en scène » la logistique, en référence à des modèles très connus en marketing expérientiel, ceux de la théâtralisation du point de vente, en jouant notamment sur des facteurs d'ambiance. Cette mise en scène ludique pourra ici présenter les ruptures en magasin comme un choix commercial performant fondé sur une logistique « coup de poing ». Ainsi, Brico Dépôt, une célèbre chaîne de distribution spécialisée dans le bricolage, a mis en place une politique d'arrivage ponctuel de produits à des prix imbattables (Rouquet \& Paché, 2017). Les produits sont disponibles en magasin en quantité limitée, et la livraison repose sur une logistique one shot. Toute la communication publicitaire adressée aux clients met l'accent sur leur indispensable vitesse de réaction : «Venez vite, il n'y en n'aura pas pour tout le monde, ne ratez pas l'occasion unique ! ».

II y a quelques années, une publicité astucieuse représentait ainsi un conteneur tombant du ciel, avec des clients fébriles de l'accueillir. En d'autres termes, alors même que Brico Dépôt a développé une politique de faible niveau de service (assumé) et de prix bas, la logistique associée aux arrivages est présentée comme étant particulièrement efficiente. D’autres exemples pourraient être présentés sur le même modèle, notamment avec une organisation scénarisée du désordre dans les magasins, accompagné de palettes traînant volontairement dans les allées : si les prix sont si faibles, nous expliquera-t-on, c'est finalement que des économies significatives sont réalisées sur le management des activités logistiques en magasin. Une théâtralisation du prix bas dont Badot (2005) a souligné toutes les vertus dans une logique de valorisation de l'infra-ordinaire.

\section{De l'ombre à la lumière}

L'importance de la logistique est rarement contestée dans sa mission de mise à disposition des produits, surtout lorsque ces produits sont aussi sensibles que des vaccins chargés de surmonter la plus importante pandémie que le monde ait eu à affronter depuis la grippe espagnole. Pourtant, la logistique n’a pas toujours bonne presse. Ce sont plutôt ses dysfonctionnements qui sont évoqués, y compris dans les grands médias. Sans doute parce que nous sommes prompts à la critique face aux trains et avions qui sont en retard, sans nous extasier face aux trains et avions qui arrivent à l'heure. Qu'une logistique fonctionne efficacement, sans bruit, est considéré comme normal, et c'est le dysfonctionnement qui dérange, conduisant à la colère et l'aigreur de clients insatisfaits par une livraison défaillante. Sans doute peut-on imaginer, en revanche, d'une image de logistique performante soit un outil majeur de rétention des clients en univers BtoB (Balmer et al., 2020).

Les logisticiennes et logisticiens, un « peuple » de l'ombre s'il en est (Paché, 2021), n'ont jamais vraiment pris conscience d'une image à améliorer de toute urgence, autrement dit de leviers à actionner pour souligner combien elles et ils sont essentiels au fonctionnement des économies de marché et, plus largement, au fonctionnement de la société. Ce travail d'investigation et de communication s'avère indispensable pour souligner que la performance de la logistique est présente, et bien présente, dans de nombreux moments de nos vies quotidiennes, du berceau au tombeau. Personne n'en doute réellement, mais trop nombreux sont celles et ceux qui l'ignorent encore. Alors même que la pandémie de Covid-19 a mis en lumière le caractère central de la logistique, y compris dans le processus délicat de vaccination de masse, il serait maladroit de ne pas en profiter pour initier la revalorisation d'une fonction support majeure. 


\section{BIBLIOGRAPHIE}

Badot, O. (2005). L'autre raison du succès de Wal-Mart : une rhétorique de l'infra-ordinaire. Revue Française du Marketing, ${ }^{\circ} 203$, pp. $97-117$.

Balmer, J., Lin, Z., Chen, W., \& He, X. (2020). The role of corporate brand image for B2B relationships of logistics service providers in China. Journal of Business Research, Vol. 117, pp. 850-861.

DeWitt, T., \& Brady, M. (2003). Rethinking service recovery strategies: the effect of rapport on consumer responses to service failure. Journal of Service Research, Vol. 6, $\mathrm{n}^{\circ}$ 2, pp. 193-207.

Halley, R. (1968). Benefit segmentation: a decision-oriented research tool. Journal of Marketing, Vol. 32, nº 3, pp. 30-35.

Hoareau, E., Ageron, B., \& Chaze-Magnan, L. (2020). Supply chain management : où sont les femmes ? The Conversation, 11 octobre.

Knemeyer, M., \& Murphy, P. (2004). Promoting the value of logistics to future business leaders. International Journal of Physical Distribution \& Logistics Management, Vol. 34, n 10 , pp. $775-792$

Koberg, E., \& Longoni, A. (2019). A systematic review of sustainable supply chain management in global supply chains. Journal of Cleaner Production, Vol. 207, pp. 1084-1098.

Lawrence, J., Lonsdale, C., \& Le Mesurier, N. (2018). Access denied? Exploring the causes of the low representation of women in senior executive positions within procurement. Journal of Purchasing \& Supply Management, Vol. 24, $n^{\circ} 4, \mathrm{pp} .304-313$.

Nasir, A. (2004). E-consumer complaints about on-line stores. Journal of Consumer Satisfaction, Dissatisfaction \& Complaining Behavior, Vol. 17, ${ }^{\circ} 1$, pp. 68-87.

Paché, G. (2021). La société malade de la Covid-19: regards logistiques croisés. Presses Universitaires d’Aix-Marseille, Aix-en-Provence.

Rajendran, S.-D., Wahab, S.-N., Ling, Y.-W., \& Yun, L.-S. (2018). The impact of logistics services on the e-shoppers' satisfaction. International Journal of Supply Chain Management, Vol. 7, $\mathrm{n}^{\circ}$ 5, pp. 461-469.

Rao, S., Goldsby, T., Griffis, S., \& lyengar, D. (2011). Electronic logistics service quality (e-LSQ): its impact on the customer's purchase satisfaction and retention. Journal of Business Logistics, Vol. 32, $\mathrm{n}^{\circ}$ 2, pp. 167-179.

Rouquet, A. (2020). La logistique du déconfinement : du juste à temps au juste écart. Management \& Data Science, Vol. 4, $n^{\circ} 4$, Article 13270.

Rouquet, A., \& Paché, G. (2017). Re-enchanting logistics: the cases of pick-your-own farm and large retail stores. Supply Chain Forum: An International Journal, Vol. 18, $\mathrm{n}^{\circ} 1$, pp. 21-29

Ruel, S., Fritz, M., \& Subramanian, N. (2020). Gender diversity for sustainability management: developing a research agenda from a supply chain perspective. Logistique \& Management, Vol. 28, n³-4, pp. 224-239

Taniguchi, E. (2014). Concepts of city logistics for sustainable and liveable cities. Procedia-Social \& Behavioral Sciences, Vol. 151 , pp. $310-317$.

Vernette, E. (1989). La segmentation par avantages recherchés, outil de stratégie marketing. Revue Française de Gestion, $\mathrm{n}^{\circ} 73$, pp. $15-22$.

Zaïdi-Chtourou, S. (2018). Effet du volume des données sur la qualité des décisions marketing. Management \& Data Science, Vol. 2, n 1 , Article 3510. 
(c) 2021 MANAGEMENT\& DATASCIENCE 